\title{
Dexmedetomidine-induced contraction of the human internal mammary artery and saphenous vein
}

\author{
Sohn JT \\ Department of Anesthesiology and Pain Medicine, Gyeongsang National University College of Medicine, Gyeongsang National \\ University Hospital, Jinju-si, Republic of Korea and Institute of Health Sciences, Gyeongsang National University, Jinju-si, \\ Republic of Korea. jtsohn@gnu.ac.kr
}

Text in PDF www.elis.sk.

To the Editor:

I have read with interest the recent article "The effects of dexmedetomidine on human internal mammary artery and saphenous vein grafts under hypothermia and normothermia" published in the Bratislava Medical Journal (1). Dexmedetomidine, a highly selective alpha-2 adrenoceptor agonist, has been widely used for sedation, analgesia, and attenuation of hemodynamic response caused by stress (2). High doses of dexmedetomidine cause hypertension due to vasoconstriction mediated via the alpha- 2 adrenoceptor stimulation of vascular smooth muscle. The following criticisms should be considered to understand fully the findings of this study. First, the magnitude of dexmedetomidine-induced contraction was expressed as a percentage of the magnitude of phenylephrine-induced contraction (1). However, using the contraction induced by potassium chloride (68 or $120 \mathrm{mM}$ ) as a reference is more appropriate, as potassium chloride-induced vessel contraction mediated by activation of voltage-gated calcium channels can be used to validate vessel viability, and furthermore, dexmedetomidine-induced contraction is mediated by calcium influx via voltage-gated calcium channels in an isolated artery $(3,4)$. Second, at least four strips of either internal mammary artery or saphenous vein should be obtained from the same patient and be assigned to four groups: endothelium-intact and normothermia, endothelium-intact and hypothermia, endothelium-denuded and normothermia, and endothelium-denuded and hypothermia. These groups would allow investigation of the effect of hypothermia on dexmedetomidine-induced contraction in an isolated vessel with or without endothelium, which is important because of interpatient variability of vasoreactivity to dexmedetomidine (3). Third, "\# $(p<0.05)$ compared to other concentrations of dexmedetomidine" in the legend of Figures 3 should be changed to "\# $(\mathrm{p}<0.05)$ compared to control (no dexmedetomidine)." Fourth,

Department of Anesthesiology and Pain Medicine, Gyeongsang National University College of Medicine, Gyeongsang National University Hospital, 15 Jinju-daero 816 beon-gil, Jinju-si, Gyeongsangnam-do 52727, Republic of Korea, and Institute of Health Sciences, Gyeongsang National University, Jinju-si, 52727, Republic of Korea

Address for correspondence: Ju-Tae Sohn, Department of Anesthesiology and Pain Medicine, Gyeongsang National University Hospital, 79 Gangnam-ro, Jinju-si, 52727, Republic of Korea

Phone: +82.55 .7508586 , Fax: +82.55 .7508142$ to analyze the effect of hypothermia and endothelial denudation on the contraction induced by dexmedetomidine in each vessel, it is more appropriate to perform statistical analysis using either twoway repeated measure analysis of variance followed by Bonferroni's comparison test or a linear mixed model. Fifth, contraction induced by dexmedetomidine is mediated by both alpha-2 adrenoceptors at low concentrations and by alpha- 1 adrenoceptors at high concentrations $(3,5)$. Thus, the question of which type of adrenoceptor is mainly involved in hypothermia-induced change of contraction induced by dexmedetomidine could be investigated by examining the effect of prazosin, an alpha-1 adrenoceptor inhibitor, and yohimbine, an alpha-2 adrenoceptor inhibitor, on the contraction induced by dexmedetomidine under both normothermia and hypothermia. Finally, the period of hypothermia used in this experiment should be described in the Methods section. I believe that this report contributes to the understanding of the impact of moderate hypothermia and endothelial denudation on dexmedetomidine-induced contraction in the isolated internal mammary artery and saphenous vein, and has important implications for the use of these vessels for coronary artery bypass graft.

\section{Reference}

1. Oc B, Arun O, Taylan SB, Oc M, Bariskaner H, Duman A. The effects of dexmedetomidine on human internal mammary artery and saphenous vein grafts under hypothermia and normothermia. Bratisl Lek Listy 2019; 120 (5): 380-385.

2. Mathur PR, Jain N, Kumar A, Thada B, Mathur V, Garg D. Comparison between lignocaine nebulization and airway nerve block for awake fiberoptic bronchoscopy-guided nasotracheal intubation: a single-blind randomized prospective study. Korean J Anesthesiol 2018; 71 (2): 120-126.

3. Yildiz O, Ulusoy HB, Seyrek M, Gul H, Yildirim V. Dexmedetomidine produces dual alpha2-adrenergic agonist and alpha1-adrenergic antagonist actions on human isolated internal mammary artery. J Cardiothorac Vasc Anesth 2007; 21 (5): 696-700.

4. Ok SH, Bae SI, Shim HS, Sohn JT. Dexmedetomidine-induced contraction of isolated rat aorta is dependent on extracellular calcium concentration. Korean J Anesthesiol 2012; 63 (3): 253-259.

5. Byon HJ, Ok SH, Lee SH, Kang S, Cho Y, Han JY, Sohn JT. Dexmedetomidine inhibits phenylephrine-induced contractions via alpha-1 adrenoceptor blockade and nitric oxide release in isolated rat aortae. Int J Med Sci 2017; 14 (2): $143-149$.

Received June 7, 2019. Accepted July 3, 2019. 